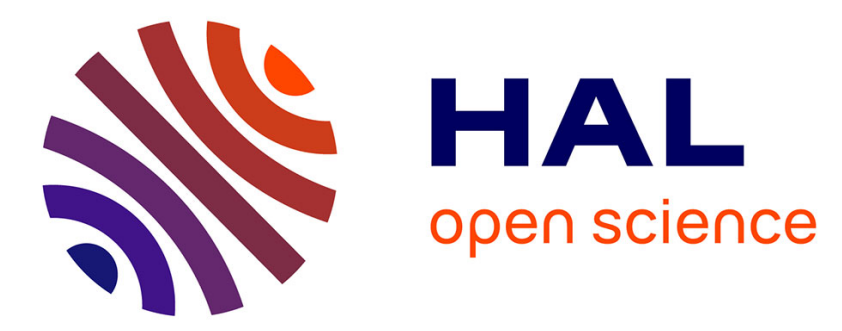

\title{
An integrative view of Luria's perspective on arithmetic problem solving: The two sides of environmental dependency
}

Jérémy Besnard, Philippe Allain, Ghislaine Aubin, Valérie Chauviré, Frédérique Etcharry-Bouyx, Didier Le Gall

\section{To cite this version:}

Jérémy Besnard, Philippe Allain, Ghislaine Aubin, Valérie Chauviré, Frédérique Etcharry-Bouyx, et al.. An integrative view of Luria's perspective on arithmetic problem solving: The two sides of environmental dependency. Journal of Clinical and Experimental Neuropsychology, 2014, 36 (1), pp.88-109. 10.1080/13803395.2013.870135 . hal-02006723

\section{HAL Id: hal-02006723 \\ https://hal.science/hal-02006723}

Submitted on 4 Jun 2021

HAL is a multi-disciplinary open access archive for the deposit and dissemination of scientific research documents, whether they are published or not. The documents may come from teaching and research institutions in France or abroad, or from public or private research centers.
L'archive ouverte pluridisciplinaire HAL, est destinée au dépôt et à la diffusion de documents scientifiques de niveau recherche, publiés ou non, émanant des établissements d'enseignement et de recherche français ou étrangers, des laboratoires publics ou privés. 


\title{
An integrative view of Luria's perspective on arithmetic problem solving: The two sides of environmental dependency
}

\author{
Jérémy Besnard $^{1,2}$, Philippe Allain ${ }^{1,2}$, Ghislaine Aubin ${ }^{1,2,3}$, Valérie Chauviré $^{1,2}$, \\ Frédérique Etcharry-Bouyx ${ }^{1,2}$, and Didier Le Gall ${ }^{1,2}$ \\ ${ }^{1}$ LUNAM Université, Laboratoire de Psychologie des Pays de la Loire (UPRES EA 4638), University of \\ Angers, France \\ ${ }^{2}$ Neuropsychological Unit, Department of Neurology, University Hospital of Angers, France \\ ${ }^{3}$ Regional Centre for Functional Rehabilitation, Angers, France
}

\begin{abstract}
Introduction: A. R. Luria was the first author to hypothesize that executive dysfunction can lead to specific deficits in arithmetic problem solving, showing that patients' performance depends on the structure of the tasks. Cummings (1995. Anatomic and behavioral aspects of frontal-subcortical circuits. Annals of the New York Academy of Sciences, 15, 1-13) proposed the term "environmental dependency" to define such behavioral disorders triggered by the characteristics of the test and pointed out also the role of executive impairments. Few studies compare executive functioning and problem solving in brain-damaged patients, and none have examined the question from this point of view. Thus, the main aim of the present paper was to study the relationship between environmental dependency and executive functions. Method: Fifty neurological patients with frontal, subcortical, and posterior brain lesions were compared to 45 matched healthy controls and were divided into two groups (dysexecutive/nondysexecutive) according to their performances on executive tasks. Then, we confronted the results of the two groups on an experimental protocol designed in accordance with Luria's proposals. We made also comparisons between groups on the basis of lesion location. Results: Our findings indicate a high association between executive functions and environmental dependency, showing that dysexecutive patients' performances were dependent on task demands. In addition, a specific frontal behavior not associated with executive functions and characterized by the solving of insoluble problems was highlighted. Conclusion: The discussion focused on the interest to take into account the methodological and clinical contributions of environmental dependency. Based on our findings and theoretical arguments, we highlight the need to fractionate this concept.
\end{abstract}

Keywords: Complex problem solving; Executive functions; Environmental dependency syndrome; Frontal lobe lesions.

Alexander Luria (1902-1977) laid the groundwork of the study of cognitive skills involved in the resolution of arithmetic problems, an area of research that remains relatively unexplored in clinical neuropsychology. Luria (1966) acknowledged the role of language comprehension in wordproblem solving, but he also suggested that executive disorders could lead to arithmetical impairments, with the description of a deficit of "regulation and control" in frontal pathology. To highlight this disorder, Luria and Tsvetkova (1967) manipulated the difficulty level of problems through the increase of the resolution algorithm complexity - that is, the number of steps needed to solve the problem. They reported that frontal patients' deficit was more obvious when the

Funding: This study received financial support from the Pays de la Loire region in France. No conflicts of interest to declare.

Address correspondence to: Jeremy Besnard, Neuropsychological Unit, Department of Neurology, University Hospital of Angers, 4, rue Larrey, 49033 Angers Cedex 01, France (E-mail: jeremy.besnard@univ-angers.fr). 
resolution of problems required a combination of several steps (e.g., 3 or 4 versus 1 or 2). It is noteworthy that the disorder affects specifically the planning and monitoring of the algorithm necessary to solve the problem, not the calculation skills - that is, the ability to make operations. To put this analysis in a more contemporary perspective, the deficit described by Luria and Tsvetkova seems to correspond to an impaired execution of "arithmetical procedures": the planning and monitoring of the sequences of steps necessary to achieve complex problems (e.g., van Harskamp \& Cipolotti, 2001). In the same vein, Luria and Tsvetkova elaborated "conflict problems," in which the operation is not isomorphic to the structure of the problem - that is, the wording of the tasks induces inverse operations from those that should be done to solve them (e.g., "Maxence has 7 apples; 2 less than Camille. How many apples has Camille?") The term "conflict" refers to the tendency to solve the problem linearly (for this example, performing the subtraction $7-2$ ). Luria and Tsvetkova argued that conflict problems necessitate the inhibition of the operation induced by the wording of the task and showed that frontal patients were more impaired to solve that kind of problem than "normal" (nonconflict) problems. In sum, Luria and Tsvetkova hypothesized that arithmetic word-problem solving may be impacted by executive impairments only, while the ability to perform calculation (e.g., addition, subtraction) can be preserved.

In other words, the authors demonstrated that frontal patients' performance is doubly dependent: (a) on the complexity of the resolution algorithm (number of steps necessary to solve the problem) and (b) on the wording of the problem (some patients were impaired only for conflict problems). This form of dependency is directly associated with the structure of the task. In that sense, it can be linked to the concept of "environmental dependency" developed by Cummings (1995). According to this author, environmental dependency could take different forms, but the common point refers to the enslavement of neurological patient's performance to external contingencies (i.e., task demands). Thus, behaviors that exhibit environmental dependency can be highlighted during traditional executive tasks like the Stroop task (patients can provide the usual response in the first part of the task but cannot inhibit it in the last part when the structure of the task changes), or the Hayling test (patients can provide a traditional response in Part A but cannot inhibit it in Part B, when an unusual response is required). From a theoretical point of view, Cummings proposed an executive interpretation of environmental dependency and argued more precisely that a failure of the "central executive component of working memory" may induce this phenomenon. As mentioned above, Luria and Tsvetkova (1967) suggested also that executive impairments (i.e., planning and inhibition) could explain the dependency to the structure of the task during complex problem solving. To clarify these assumptions, it seems necessary to give a more precise definition of executive functions. Although there is no consensus in the literature, two main theoretical approaches have guided the development of the concept of executive functions. The first derives directly from the seminal works of Luria (1966) and defines processes like planning, inhibition, shifting, and action initiation as "executive" (Lezak, 1982). In this perspective, Norman and Shallice (1986) have designed a cognitive model of action control in which a high-level process (the supervisory attentional system; SAS) underlies these functions. The second approach refers to Baddeley and Hitch's theory (1974) of working memory and postulates that a central executive is involved in different processes like inhibition, task coordination, shifting, and updating. Interestingly, Baddeley (1986) made the link with Norman and Shallice's proposals by acknowledging that the functions of the central executive correspond to an adequate approximation of the processes of the SAS. A more contemporary definition of executive functions is provided by Godefroy (2003; see also Godefroy et al., 2010), who tried to draw up a list of executive processes underlain by both the central executive and the SAS. The authors proposed that deficits of inhibition, planning, shifting, rule detection, clustering, and task coordination are the main cognitive disorders suggestive of dysexecutive syndrome. This is the definition we adopted in our study. In sum, it appears that Cummings (1995) and Luria and Tsvetkova (1967) share the same point of view concerning the theoretical interpretation of environmental dependency, in terms of an executive disruption.

In the domain of problem solving, some authors have pointed out that impairments in executive functioning can lead to secondary deficits in arithmetic processing, but these studies have been mainly conducted in the developmental literature (e.g., Lee, Lynn Ng, \& Fong Ng, 2009). In fact, few works have tried to examine specifically the role of executive functions in arithmetic problem solving with a neuropsychological approach, and none considers these deficits under the perspective of environmental dependency. In patients with frontal lobe damage, several publications focused on the calculation deficit in complex multistep arithmetic problems. However, most studies 
describe a deficit in the retrieval of arithmetical facts (e.g., table facts like $7 \times 8$ ), not a selective impairment in executing complex procedures (e.g., Semenza, Miceli, \& Girelli, 1997). In case of basal ganglia dysfunction, impairments in arithmetic problem solving have been recently associated with executive deficits (Delazer et al., 2004; Roşca, 2009; Zamarian et al., 2009; Zamarian et al., 2006). However, as far as we are aware, there is only one group study (Zamarian et al., 2006), which demonstrates no difference between patients with Parkinson's disease and normal controls on tests assessing arithmetic abilities. In addition, calculation deficits were demonstrated in patients with posterior brain damage (e.g., Dehaene \& Cohen, 1997), but the impact of executive impairments on problem solving is not investigated while posterior lesions can also induce executive disruption (e.g., Colette \& Van der Linden, 2002). Furthermore, to the best of our knowledge, there is only one study after Luria and Tsvetkova (1967) in which the authors designed the methodology to assess specifically the role of executive functions in arithmetic processes (Zamarian, Semenza, Domahs, Benke, \& Delazer, 2007). They compared patients with Alzheimer's disease and mild cognitive impairment (MCI) on computerized arithmetic tasks, which one of them was designed to assess specifically the ability to inhibit automatic responses ("Stroop-like" condition). Interestingly, the authors showed that there was a correlation between the Stroop test and the measure of interference effects given by the Stroop-like condition.

A few years ago, another disorder during complex problem solving was described, which seems to be associated with environmental dependency (Aubin, Le Gall, \& Guyard, 1994; Le Gall, Allain, \& Aubin, 2001; Le Gall, Aubin, Allain, \& Guyard, 1993). In the wake of Luria and Tsvetkova (1967), the authors examined the behavior of frontal patients when insoluble problems were included (e.g., "In a boat, there are 160 passengers. 100 fall into the sea. How old is the captain?"). In these proposals, the text looks like a standard problem, but there is an inconsistency between data and question. With a protocol including both soluble and insoluble problems, the authors found two kinds of results in frontal pathology. Some patients were impaired to solve soluble problems but they could reject insoluble items, while others showed an inability to challenge irrelevant propositions, which results in the solving of insoluble problems (e.g., "160 - 100; the captain was 60 years old"). Interestingly, these patterns of performance were dissociated for certain patients: some of them were able to solve normal problems but also proposed solutions for insoluble problems; while others demonstrated the inverse profile. This means that the solving of insoluble problems seems not to be attributable to a global comprehension deficit and can be reported without impairment in calculation. These studies have been mainly conducted in the frontal pathology (Aubin et al., 1994; Le Gall et al., 2001; Le Gall et al., 1993), but one work with 10 patients with Huntington's disease revealed that the solving of insoluble problems was not observed (Allain et al., 2005). Thus, additional studies are needed to clarify the frontal specificity of this disorder and shed light on the issue of the potential link with Cumming's (1995) concept of environmental dependency.

Thus, the aim of the present study was first to clarify the executive interpretation of environmental dependency, using a protocol of complex arithmetic problems inspired by Luria's perspective. Since the theoretical publications of Luria and Tsvetkova (1967) and Cummings (1995), no study has been carried out to support this theoretical assumption with empirical data. Second, this objective allows us at the same time to make some contribution to the issue of the relationship between executive functions and complex problem solving, as several authors have emphasized the need thereafter (McNeil \& Burgess, 2002; Semenza et al., 1997; Zamarian et al., 2007; Zamarian et al., 2006). Third, we want to provide more empirical data about the behavior described in previous publications (the solving of insoluble problems; Aubin et al., 1994; Le Gall et al., 2001; Le Gall et al., 1993), especially for the frontal specificity of the phenomenon and for the potential link with Cumming's concept of environmental dependency. Demonstrating that the solving of insoluble problems is a pathognomonic behavior of frontal pathology seems an important issue, because it would provide a substantial contribution to the distinction between "executive" and "frontal" syndromes. As some authors pointed out, the terms "frontal syndrome" and "dysexecutive syndrome" are often used interchangeably but are not superimposable (e.g., Anderson, Levin, \& Jacobs, 2002; Burgess, Alderman, Volle, Benoit, \& Gilbert, 2009). Thus, dysexecutive syndrome does not allow a fully satisfactory explanation of some behavioral disorders associated with frontal pathology. Moreover, as Stuss (2007) emphasized, it appears necessary to propose methodologies suitable for dissociate "executive" and "frontal" syndromes by highlighting specific frontal disorders to better contribute to the comprehension of frontal lobe syndrome. 
Several hypotheses are proposed. (a) Following the assumption of Luria and Tsvetkova (1967) and in reference to the executive interpretation of environmental dependency (Cummings, 1995), we hypothesized that executive impairments should lead to an enslavement of the performances to task demands in the context of arithmetic problem solving. This means that patients with weak performances on executive tests (dysexecutive patients) should be more impaired than nondysexecutive patients for conflict problems and when the level of algorithm resolution increases - that is, the number of steps necessary to solve the problem. Consequently, the total number of problems failed will be higher for dysexecutive patients. Following the assumptions of several authors (McNeil \& Burgess, 2002; Semenza et al., 1997; Zamarian et al., 2007; Zamarian et al., 2006), who stressed the link between executive functions and complex problem solving, we also posit that (b) some correlations between scores on executive tasks and performances in problem solving should be revealed. More generally, we hypothesized that patients with weak performances on solving complex problems should be more impaired on executive tasks than patients who show few or no arithmetic deficits. Differences between groups will allow us to highlight the executive processes involved. Finally, if the solving of insoluble problems is specifically associated with frontal lobe damage (Allain et al., 2005; Aubin et al., 1994; Le Gall et al., 2001; Le Gall et al., 1993), we hypothesized that (c) frontal patients should be more likely to propose solution for this kind of problem than those with subcortical or posterior brain damages. This assertion means that executive processes should be not or less implicated in this particular behavior.

\section{METHOD}

\section{Participants}

A total of 95 participants took part in the present study: 50 brain-injured patients admitted to the Neurological Department of the University Hospital or to the Regional Centre for Functional Rehabilitation, and 45 control participants recruited from local associations, ranging between 20 and 76 years of age.

\section{Neurological patients}

None of the patients showed impaired language comprehension, or signs of aphasia or alexia, assessed using the "Protocole Montréal-Toulouse
d'Examen Linguistique de l'Aphasie" (MT-86; Beland \& Giroud, 1992) or the "Boston Diagnostic Aphasia Examination" (BDAE; Mazaux \& Orgogozo, 1981). This inclusion criterion was necessary given the fact that the material was constituted of word arithmetic problems. Exclusion criteria for participant selection were also motor impairments that would prevent participation (written responses), previous history of psychiatric disorders, neurological illness, or clinically significant depression. Using computed tomography/magnetic resonance imaging scans closest to the time of testing, patients were divided into three groups: a frontal group composed of 20 patients with frontal lobe lesion, a subcortical group $(n=$ $20)$, and a posterior group $(n=10)$ in whom the frontal lobe was spared. Clinical magnetic resonance imaging/computed tomography (MRI/CT) scans were available for 19 of 20 frontal patients, 9 of 10 posterior patients, and 17 of 20 patients with subcortical lesions. There were neuroimaging reports for all patients. Topography of the lesions and main characteristics of patients are presented in Table 1. Imaging findings were evaluated by two experienced neurologists (V.C. and F.E.-B.), who were blinded to the cognitive performance of the patients. Lesion location was conducted according to the procedure of Damasio and Damasio (1989), which maps brain lesions onto standard templates. If the lesion involved both frontal and nonfrontal areas, the patient was classified as frontal. Presence of lesions involving both frontal and nonfrontal areas was not an exclusion criterion, given the fact that we compared three groups of patients. In the frontal group, the etiology of the lesions included vascular $(n=5)$, traumatic $(n=11)$, and degenerative $(n=3)$ causes. One patient sustained carbon monoxide intoxication. The posterior group consisted of 10 patients (six with lesions due to focal stroke, two due to trauma, one patient had temporal atrophy of unknown origin, and one had a tumor). The patients with subcortical cerebral lesions suffered from Parkinson's disease $(n=8)$, Huntington's disease $(n=4)$, focal stroke $(n=5)$, progressive supranuclear palsy $(n=1)$, leukoencephalopathy $(n=1)$, and Leigh's disease $(n=1)$.

\section{Control subjects}

The control group was constituted of 45 healthy subjects (32 women and 13 men). Patients and control participants were matched on typical demographic criteria (age, education level), except for gender, chi-square test, $\chi^{2}(3, N=95)=17.01$, $p<.001$. Given the importance of education level in arithmetic performances, we conducted post hoc 
TABLE 1

Topographies of the lesion and main characteristics of patients

\begin{tabular}{|c|c|c|c|c|c|c|c|c|c|}
\hline Patient & Group & Gender & $\begin{array}{l}\text { Age } \\
\text { (years) }\end{array}$ & $\begin{array}{l}\text { Education } \\
\text { (years) }\end{array}$ & Etiology & $\begin{array}{c}\text { Time since } \\
\text { injury (months) }\end{array}$ & $\begin{array}{c}\text { Topography of the } \\
\text { lesion }\end{array}$ & $\begin{array}{l}\text { Side of } \\
\text { the lesion }\end{array}$ & $M M S E$ \\
\hline F1 & Frontal & M & 72 & 9 & Dementia syndrome & & $\begin{array}{l}\text { Fronto-parietal } \\
\text { atrophy }\end{array}$ & Bilateral & 25 \\
\hline $\mathrm{F} 2$ & Frontal & $\mathrm{F}$ & 63 & 15 & $\begin{array}{l}\text { Fronto-temporal } \\
\text { dementia }\end{array}$ & & $\begin{array}{l}\text { Fronto-temporal } \\
\text { atrophy }\end{array}$ & Bilateral & 18 \\
\hline F3 & Frontal & M & 50 & 8 & Trauma & 2 & Fronto-temporal & Bilateral & 27 \\
\hline $\mathrm{F} 4$ & Frontal & $\mathrm{F}$ & 76 & 14 & Stroke & 13 & Frontal & Left & 29 \\
\hline F5 & Frontal & M & 69 & 9 & Intoxication & 26 & $\begin{array}{l}\text { Fronto-temporo- } \\
\text { occipital }\end{array}$ & Bilateral & 28 \\
\hline F6 & Frontal & M & 53 & 9 & Trauma & 12 & Frontal & Left & 23 \\
\hline F7 & Frontal & M & 76 & 9 & Trauma & 2 & Frontal & Bilateral & 24 \\
\hline F8 & Frontal & M & 72 & 9 & Dementia syndrome & & Frontal atrophy & Bilateral & 25 \\
\hline F9 & Frontal & M & 50 & 9 & Trauma & 34 & Fronto-temporal & Bilateral & 30 \\
\hline F10 & Frontal & M & 38 & 9 & Stroke & 8 & Fronto-parietal & Bilateral & 24 \\
\hline F11 & Frontal & M & 37 & 12 & Trauma & 15 & $\begin{array}{c}\text { Frontal and } \\
\text { cingulate }\end{array}$ & Bilateral & 28 \\
\hline F12 & Frontal & M & 26 & 8 & Trauma & 1 & Frontal & Bilateral & 28 \\
\hline F13 & Frontal & M & 54 & 8 & Trauma & 1 & Fronto-temporal & Bilateral & 23 \\
\hline F14 & Frontal & M & 18 & 9 & Trauma & 1 & $\begin{array}{l}\text { Fronto-temporo- } \\
\text { occipital }\end{array}$ & Bilateral & 29 \\
\hline F15 & Frontal & M & 24 & 9 & Trauma & 1 & Frontal and insula & Right & 28 \\
\hline F16 & Frontal & M & 52 & 9 & Trauma & 8 & Fronto-temporal & Bilateral & 17 \\
\hline F17 & Frontal & M & 20 & 11 & Trauma & 3 & $\begin{array}{l}\text { Fronto-temporo- } \\
\text { parietal }\end{array}$ & Bilateral & 27 \\
\hline F18 & Frontal & M & 60 & 7 & Stroke & 1 & Frontal & Bilateral & 20 \\
\hline F19 & Frontal & $\mathrm{F}$ & 73 & 9 & Stroke & 2 & $\begin{array}{l}\text { Fronto-temporo- } \\
\text { parieto-occipital }\end{array}$ & Right & 23 \\
\hline $\mathrm{F} 20$ & Frontal & $\mathrm{F}$ & 69 & 8 & Stroke & 2 & Frontal & Bilateral & 24 \\
\hline $\mathrm{SC} 1$ & Subcortical & M & 50 & 9 & Huntington disease & & - & & 26 \\
\hline $\mathrm{SC} 2$ & Subcortical & M & 39 & 9 & Huntington disease & & - & & 26 \\
\hline $\mathrm{SC} 3$ & Subcortical & $\mathrm{F}$ & 51 & 8 & PSP & & - & & 23 \\
\hline $\mathrm{SC} 4$ & Subcortical & M & 42 & 10 & Huntington disease & & - & & 24 \\
\hline $\mathrm{SC} 5$ & Subcortical & M & 65 & 5 & Stroke & 1 & $\begin{array}{l}\text { Subcortical } \\
\text { atrophy }\end{array}$ & Bilateral & 22 \\
\hline SC6 & Subcortical & $\mathrm{F}$ & 60 & 6 & Huntington disease & & - & & 30 \\
\hline SC7 & Subcortical & $\mathrm{F}$ & 18 & 11 & Stroke & 2 & Thalamic & Bilateral & 28 \\
\hline $\mathrm{SC} 8$ & Subcortical & $\mathrm{F}$ & 28 & 14 & Stroke & 1 & Thalamic & Left & 30 \\
\hline SC9 & Subcortical & $\mathrm{F}$ & 36 & 15 & Stroke & 1 & Thalamic & Bilateral & 27 \\
\hline $\mathrm{SC} 10$ & Subcortical & M & 77 & 6 & Parkinson disease & & - & & 26 \\
\hline $\mathrm{SC} 11$ & Subcortical & M & 62 & 14 & Stroke & 1 & Thalamic & Bilateral & 26 \\
\hline $\mathrm{SC} 12$ & Subcortical & $\mathrm{M}$ & 46 & 9 & Parkinson disease & & - & & 29 \\
\hline $\mathrm{SC} 13$ & Subcortical & M & 57 & 12 & Parkinson disease & & - & & 21 \\
\hline $\mathrm{SC} 14$ & Subcortical & M & 26 & 9 & Leigh disease & & $\begin{array}{l}\text { Putamen and } \\
\text { caudate nucleus }\end{array}$ & Bilateral & 26 \\
\hline $\mathrm{SC} 15$ & Subcortical & $\mathrm{F}$ & 84 & 6 & Parkinson disease & & - & & 25 \\
\hline $\mathrm{SC} 16$ & Subcortical & $\mathrm{F}$ & 72 & 9 & Parkinson disease & & - & & 29 \\
\hline $\mathrm{SC} 17$ & Subcortical & $\mathrm{F}$ & 65 & 6 & Parkinson disease & & - & & 30 \\
\hline $\mathrm{SC} 18$ & Subcortical & M & 71 & 15 & Parkinson disease & & - & & 27 \\
\hline $\mathrm{SC} 19$ & Subcortical & $\mathrm{F}$ & 73 & 6 & Parkinson disease & & - & & 25 \\
\hline $\mathrm{SC} 20$ & Subcortical & M & 52 & 9 & Leukoencephalopathy & & Striatum & Left & 28 \\
\hline P1 & Posterior & M & 50 & 12 & Stroke & 2 & Temporo-parietal & Left & 26 \\
\hline $\mathrm{P} 2$ & Posterior & $\mathrm{F}$ & 49 & 12 & Stroke & 2 & Temporal & Left & 26 \\
\hline P3 & Posterior & M & 30 & 12 & Stroke & 1 & $\begin{array}{l}\text { Temporo-parieto- } \\
\text { occipital }\end{array}$ & Left & 29 \\
\hline P4 & Posterior & M & 28 & 9 & Trauma & 4 & Temporal & Left & 27 \\
\hline P5 & Posterior & M & 20 & 9 & Trauma & 1 & Temporal & Left & 30 \\
\hline P6 & Posterior & $\mathrm{F}$ & 53 & 9 & Meningioma & 1 & Temporal & Left & 25 \\
\hline P7 & Posterior & M & 56 & 16 & Stroke & 1 & Temporal & Left & 28 \\
\hline P8 & Posterior & M & 48 & 9 & Stroke & 1 & Temporal & Right & 23 \\
\hline P9 & Posterior & M & 77 & 12 & Stroke & 3 & Occipital & Left & 26 \\
\hline P10 & Posterior & $\mathrm{F}$ & 60 & 9 & Degenerative disease & & Temporal & Bilateral & 28 \\
\hline
\end{tabular}

Note. $\mathrm{M}=$ male $\mathrm{F}=$ female. $\mathrm{MMSE}=$ Mini-Mental State Examination; $\mathrm{PSP}=$ progressive supranuclear palsy. 
comparisons between groups. As can be seen in Table 2, there was no significant difference for this variable. All participants had normal or corrected-to-normal vision. All subjects gave their informed consent to take part in this study, which was approved by the local research ethics committee and was conducted in compliance with the Helsinki Declaration.

\section{Neuropsychological and clinical assessment}

\section{Neuropsychological assessment}

The dysexecutive syndrome was assessed with seven tasks, in accordance with the definition of executive functions proposed by Godefroy (2003; Godefroy et al., 2010). These tasks are part of the GREFEX protocol (Azouvi et al., 2001) and are frequently used for the assessment of executive functions. The Stroop interference task (Stroop, 1935) was given to measure response inhibition, as well as the number of perseverative errors on the Modified Card Sorting Test (MCST; Nelson, 1976). The numbers of correct series and errors on the MCST give also information about rule detection, as well as the performance on the Brixton task (Burgess \& Shallice, 1996). The Trail Making Test (TMT; Lezak, 1983) was given to assess cognitive shifting. Participants were also tested on clustering (categorical and verbal phonological fluency; animals and p-words; Cardebat, Doyon, Puel, Goulet, \& Joanette, 1990), planning (Six Elements task of the Behavioural Assessment of the Dysexecutive Syndrome, BADS; Wilson, Alderman, Burgess, Emslie, \& Evans, 1996). Task coordination was assessed with the Dual Task of Baddeley, with the mu index to quantify the dual task decrement (Baddeley, Della Sala, Papagno, \& Spinnler, 1997). This task is also frequently mentioned for the assessment of the processes of the central executive of Baddeley and Hitch's (1974) theory (e.g., Roussel, Dujardin, Hénon, \& Godefroy, 2012). Interestingly, a recent normalization of the GREFEX protocol (Godefroy et al., 2010; Roussel \& Godefroy, 2008) allows determination of whether the patient showed a dysexecutive syndrome or not, on the basis of the number of pathological performances on standard criteria of the tasks. Thus, according to the normative data for each criteria (e.g., time, number of errors), the diagnosis of dysexecutive syndrome can be made if the patient showed a deficit for at least three criteria, all tests combined (for instance, pathological number of errors on the Stroop and the Brixton tasks and pathological number of correct series on the MCST). Table 2 outlines the criteria taken into account for the GREFEX protocol. Participants performed also the Mini Mental State Examination (Folstein, Folstein, \& McHugh, 1975; see Tables 1 and 2).

Clinical measures of working memory consisted of the Digit Span subtest of the Wechsler Adult Intelligence Scale-Third Edition (WAIS-III; Wechsler, 1997). In Digit Span forward, participants heard a sequence of digits and were required to repeat the sequence in the exact order it was presented. The number of digits in the sequence started with two digits and increased progressively. The raw score was the number of correct trials. Digit Span forward is a measure of the verbal storage-only component of working memory (phonological loop component of Baddeley's multicomponent model of working memory; see, for example, Bon, Belliard, Eustache, \& Desgranges, 2009; Samson, 2006). For Digit Span backward, participants heard also a sequence of digits, but they were required to repeat them in reverse order. The sequences became increasingly more difficult (from a sequence of two digits to a maximum of nine digits). The raw score of Digit Span backward was the number of correct trials. Digit Span backward is a measure of the storage and processing components of verbal working memory (i.e., phonological loop plus central executive components of working memory; see Bon et al., 2009; Samson, 2006).

\section{Complex arithmetic problems}

We designed a protocol of arithmetic problems organized into a hierarchy of difficulty levels, through the increasing complexity of the resolution algorithm. In accordance with the proposals of Luria and Tsvetkova (1967) and following Fasotti (1992), three levels of difficulty have been proposed, divided into two degrees (with or without conflict). Twelve problems have been made: four problems per difficulty level. For each level, problems were divided into two categories: two nonconflict problems and two conflict problems (the wording of the problem induces others operations than those necessary to solve it). All problems were related to a quantity. Level 1 was composed of four problems constructed following the structure $a+(a-b)=x$, whose algorithm resolution requires two operations (nonconflict problem: "Pierre has 20 euros; his brother has 5 euros less. How many euros do they have together?"; conflict problem: "Maxence has 7 apples, 2 less than Camille. How many apples do they have together?"). The second level included four problems whose resolution needs three steps, 


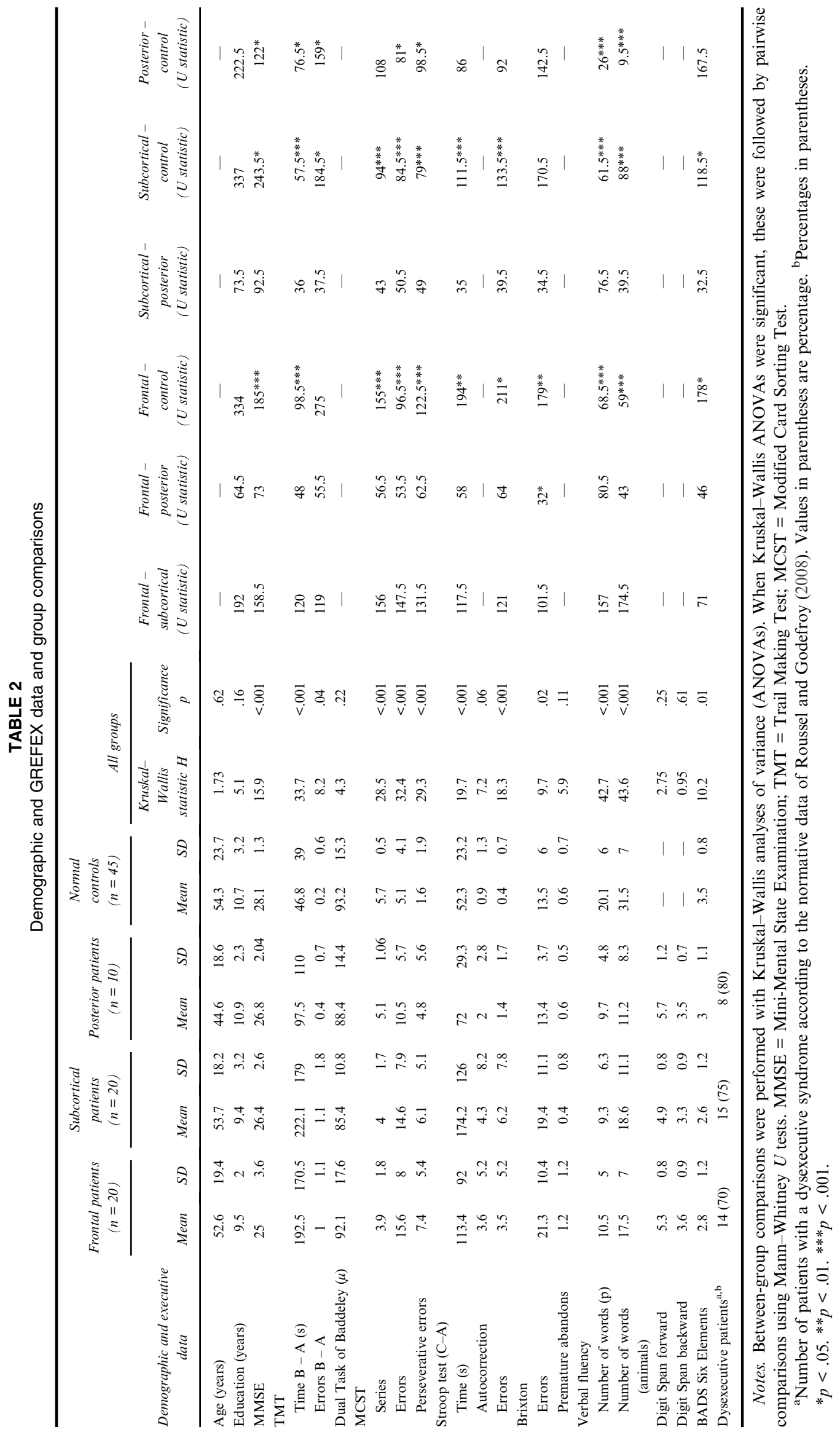


according to the algorithm $a+(a-b)+(a+c)=x$ (nonconflict problem " $A$ non-smoking rail wagon contains 50 passengers. In the smoking wagon, there are 30 passengers less than in the non-smoking wagon. In the dining wagon, there are 15 passengers more than in the non-smoking wagon. How many passengers are present in these three wagons in total?" and conflict problem: "Jerome has 22 marbles, 3 less than Stephanie. Sophie has 6 marbles less than Jerome. How many marbles do they have together?"). Level 3 was composed of four problems elaborated according to the algorithm $a+$ $(a-b)+\{[a+(a-b)]+c\}=x$, whose solving necessitates four steps. The following are examples of a simple problem: "In the A bouquet, there are 19 flowers. In the $B$ bouquet, there are 7 flowers less than in the A bouquet. The bouquet $C$ has 8 flowers more than in the bouquets $A$ and $B$ together. How many flowers are there in total?" and of a conflict problem: "In the port of Le Havre, there are 340 boats; 70 more boats than the port of Brest. The port of Dieppe has 100 boats less than in these two ports together. How many boats are there in total?" The operations required to solve each problem are outlined in Appendix A.

To highlight the behavior described in previous works (Aubin et al., 1994; Le Gall et al., 2001; Le Gall et al., 1993), six insoluble problems were also administered. We varied the numerical plausibility for half of the insoluble problems (the opportunity to perform a simple operation for achieving a result consistent with the question), while maintaining the semantic coherence (each element of the wording is compatible with each other). An example of a plausible insoluble problem is: "On a boat, there are 106 passengers in second class and 50 in first class. How old is the captain?" The problem is plausible because it is conceivable to make a subtraction $(106-50)$ to reach a result corresponding to an order of magnitude for an age. An example of non-plausible insoluble problem is: "Ms. Durand bought 3 steaks and 4 fillets of beef at the butcher shop. How old is her husband?" Here the problem is not plausible because it seems more difficult to reach a consistent result for an age by making operations with the data. For all insoluble problems, the question was related to the age of a person. If the patient asks whether there are some irrelevant problems, the examiner simply said "It is possible that some problems have no solutions; it is up to you to determine the relevance of the problem and solve it or not." All problems were presented on 6 sheets of A4 paper ( 3 problems per page), in a randomized order. For each of the 18 problems, we asked subjects to read the problem aloud and then to write all the operations required for the resolution on the sheet. To reduce the charge of working memory, the participant could read the problem as many times as desired. The assessment was not limited in time and could be made in several sessions, depending on the fatigability of the patient. At the beginning, an example of a two-step word problem (the lowest level) was proposed.

\section{Analysis of responses}

The analysis of participants' responses was conducted following quantitative and qualitative approaches.

Quantitative approach. For the 12 soluble problems, "total failure" score refers to the number of failed problems (maximum score equal to 12 for this criterion). Based on written responses of participants, we identify four types of failures. (a) The first type refers to a "calculation failure"; the subject develops a correct algorithm to solve the problem but failed in performing one or more operations. (b) The second kind of failure refers to the difficulty to achieve the correct resolution algorithm ("algorithm failure"). Results of calculations are correct, but the different steps of the resolution algorithm are inadequate or incomplete. The omission of an operation has been listed in this second type of failure. (c) The third type of failure ("conflict failure") is the solving of a conflict problem according to the operation induced by the wording of the task. Appendix B shows examples of algorithm and conflict failure. In accordance with the proposals of Luria and Tsvetkova (1967), an "executive" failure score was obtained by summing "algorithm" and "conflict" failures. When several errors were made for a same problem (e.g., calculation and algorithm), we rated this result as "joint failure" and coded it, but the first kind of failure only was taken into account for the statistical analysis (the primary cause of failure) to obtain a maximum score of failure equal to 12 .

For each level of problem, the number of failures was reported according to this analysis grid as well as the total number of failures. Given that a "calculation failure" can be due to several calculation errors during the solving of one problem (contrary to conflict or algorithm failures), we recorded also the total number of calculation errors. For insoluble problems, a score of resolution was based on the number of items for which the participant proposed a solution by manipulating the data. The errors were assessed by two independent judges, one of whom was blind to the results of the cognitive tasks. Interrater reliability was calculated 
using Spearman rank-order correlation coefficients (Spearman's rho) between number of calculation failure, algorithm failure, conflict failure, calculation errors, and insoluble problems solved. Spearman rank order correlations were high for all variables taken into account $(.83<\rho<1)$, indicating excellent correlations (e.g., Campos et al., 2009).

Qualitative approach. Qualitative rating consisted in recording the attitudes and verbalizations of participants confronted with insoluble problems. Four kinds of behavior have been identified: (a) rejection: The subject immediately detects the incongruous nature of the problem and rejects it without hesitation; (b) partial resolution: Some problems are solved (below a cutoff score), but then the participant hesitates and rejects the following items, saying that he uncovered the aberrant nature of the problems; (c) resolution not argued: The number of insoluble problems solved is greater than the pathological threshold (cutoff score), but no justification is provided, and the plausibility of the answer is not taken into account; the subject manifests some incomprehension and challenges the proposals of the examiner; (d) reasoned resolution: The number of insoluble problems solved is beyond the cutoff score, and the subject tries to provide consistent results for an age by manipulating the data, while arguing his answers, without criticizing the nature of the problems. Depending on the availability of the recording material, some patients were filmed to facilitate the scoring.

\section{Statistical analysis}

Given the unequal group sizes, unequal variances and the significant deviation of the dataset to normal distribution, nonparametric tests were employed. Between-group comparisons were performed using Kruskal-Wallis one-way analyses of variance (ANOVAs). When Kruskal-Wallis ANOVAs were significant, these were followed by post hoc comparisons using Mann-Whitney $U$-tests. Spearman rank order correlations were used to study relationships between variables. The alpha level was selected at $p<.05$. The cutoff scores were established based on the performance of the group of normal subjects, using the percentile method (Efron, 1987; Efron \& Tibshirani, 1993). The threshold values were positioned at the 95th percentile of the performances of the control group and were obtained for the resolution score of insoluble problems (1.9 rounded to 2) and the resolution score of soluble problems $(5.25$ rounded to 5 ; i.e., number of failures $\leq 7$ ). The different subgroups of patients were constituted from these cutoff scores.

\section{RESULTS}

\section{Neuropsychological characteristics}

\section{Executive data}

First, we compared the four groups of participants on executive criteria. As shown in Table 2, frontal and subcortical patients were impaired on all of the criteria, except for the number of errors on the TMT for frontal patients and on the Brixton task for subcortical patients. Patients with posterior brain damage performed significantly worse than control subjects on the MCST (numbers of errors and perseverative errors) and verbal fluency. They were also slower than healthy controls on the TMT. Overall, frontal and subcortical patients demonstrated impairment for $11 / 12$ $(91.6 \%)$ executive criteria, whereas posterior patients were deficient for $6 / 12(50 \%)$ variables. All groups of patients had a lower score than controls on the Mini Mental State Examination (MMSE). The performances of neurological patients were superimposable for executive tests, except for the number of errors on the Brixton task between frontal and posterior patients, Mann-Whitney $U=32, p=.02$. According to the norms of the executive protocol (Godefroy et al., 2010; Roussel \& Godefroy, 2008), 14 frontal patients $(70 \%), 15$ subcortical patients $(75 \%)$, and 8 $(80 \%)$ patients with posterior brain damage were classified as "dysexecutive" (three or more pathological performances for the criteria taken into account for each task). This difference is not significant, chi-square test, $\chi^{2}(2, N=37)=0.36, p=$ .83. In addition, there was no difference between groups for measure of working memory (Digit Span subtests of the WAIS-III).

\section{Problem-solving results}

\section{Comparisons of performances on soluble problem solving}

To examine the first hypothesis, comparisons were conducted between patients categorized as "dysexecutive" and "nondysexecutive." Thirteen $(26 \%)$ patients were classified as "nondysexecutive," while they were $37(74 \%)$ with a dysexecutive syndrome. There was no difference between the groups for education level $(U=215.5, p=.67)$. As can be seen in Table 3, difference between the two groups 
TABLE 3

Group comparison between dysexecutive and nondysexecutive patients for the results for arithmetic protocol

\begin{tabular}{|c|c|c|c|c|c|c|c|c|}
\hline \multirow[b]{3}{*}{ Results } & \multirow{2}{*}{\multicolumn{2}{|c|}{$\begin{array}{l}\text { Normal } \\
\text { controls }\end{array}$}} & \multirow{2}{*}{\multicolumn{2}{|c|}{$\begin{array}{c}\text { Dysexecutive } \\
\text { patients }\end{array}$}} & \multirow{2}{*}{\multicolumn{2}{|c|}{$\begin{array}{c}\text { Non-dysexecutive } \\
\text { patients }\end{array}$}} & \multirow{2}{*}{\multicolumn{2}{|c|}{$\frac{\text { Group comparison }}{\text { Dysexecutive -nondysexecutive }}$}} \\
\hline & & & & & & & & \\
\hline & $M$ & $S D$ & $M$ & $S D$ & $M$ & $S D$ & $U$ statistic & Significance $p$ \\
\hline Education (years) & 10.7 & 3.2 & 9.7 & 2.8 & 9.9 & 2.13 & 215.5 & .67 \\
\hline \multicolumn{9}{|l|}{ Mean number of failures ${ }^{a}$} \\
\hline Total & 2.9 & 2.2 & 6.1 & 3.1 & 4.2 & 3.1 & 146 & .045 \\
\hline Level 1 & 0.4 & 0.6 & 1.1 & 1.2 & 1 & 0.9 & 201.5 & .73 \\
\hline Level 2 & 0.9 & 0.9 & 2.1 & 1.4 & 1.4 & 1.4 & 169 & .14 \\
\hline Level 3 & 1.5 & 1.3 & 2.8 & 1.1 & 1.8 & 1.3 & 126.5 & .011 \\
\hline Executive failure $^{\mathrm{b}}$ & 2.3 & 2.1 & 4.7 & 3 & 2.7 & 2.6 & 126 & .013 \\
\hline Calculation failure & 0.57 & 0.7 & 1.5 & 1.3 & 1.5 & 1.8 & 212 & .61 \\
\hline \multicolumn{9}{|c|}{ Mean number of problems solved ${ }^{\mathrm{c}}$} \\
\hline Conflict problems & 4.4 & 1.5 & 2.5 & 2 & 3.7 & 1.9 & 149.5 & .052 \\
\hline Nonconflict problems & 4.6 & 1 & 3.4 & 1.5 & 4.1 & 1.7 & 156.5 & .08 \\
\hline Insoluble problems & 0.3 & 1 & 1.2 & 2.1 & 1.7 & 2.6 & 223.5 & .81 \\
\hline Calculation errors $^{\mathrm{d}}$ & 0.57 & 0.7 & 2.1 & 1.8 & 1.9 & 1.8 & 213.5 & .64 \\
\hline
\end{tabular}

Notes. Between-group comparisons were performed using Mann-Whitney $U$ tests.

aMaximum score equal to 12 for "total," "executive failure," and "calculation failure"; maximum score equal to 4 for "Level 1," "Level 2," and "Level 3. 'Executive failure = sum of algorithm and conflict failures. "Maximum score equal to 6 for "conflict," "nonconflict," and "insoluble" problems. ${ }^{\mathrm{d}}$ Total number of calculation errors.

was highlighted for the total number of problems failed, Mann-Whitney $U=146, p=.045$. The two groups differed only on the number of executive failures, Mann-Whitney $U=126, p=013$, but not on the number of calculation failures, MannWhitney $U=212, p=.61$, or the total number of calculation errors, Mann-Whitney $U=213.5, p=$ .64. Interestingly, there was no difference for the numbers of Level 1 problems failed, MannWhitney $U=201.5, p=.73$, and Level 2 problems failed, Mann-Whitney $U=169, p=.14$, but only for the number of Level 3 problems failed, MannWhitney $U=126.5, p=.011$. Moreover, the number of conflict problems correctly solved approached significance, Mann-Whitney $U=$ $149.5, p=.052$, but there was no difference between the two groups for the number of nonconflict problems, Mann-Whitney $U=156.5, p=.08$, and the number of insoluble problems for which a solution was given, Mann-Whitney $U=228, p=.78$.

\section{Relationship between arithmetic and cognitive performances}

To explore the second hypothesis, Spearman correlations were carried out between the experimental data obtained from patients and executive criteria. We studied the relationship between performance on executive tasks, calculation and executive failures, calculation errors, and insoluble problem scores. Table 4 gives an overview of these analyses. There were significant correlations between calculation failure and five variables $(35.7 \%)$ of the executive protocol, which included: (a) measures of inhibition (completion time and number of errors in the Stroop task); (b) planning (Six Elements subtest of the BADS); (c) clustering (verbal letter fluency); and (d) rule detection (number of errors in the Brixton task). The strongest correlation was found between calculation failure and the Six Elements score, $\rho=-.57$. The same correlations have been highlighted between total number of calculation errors and executive scores, with two additional significant coefficients (shifting: number of errors in the TMT, $\rho=.41$; rule detection: number of correct series in the MCST, $\rho=-.29$ ). Numerous correlations were uncovered between executive scores and the number of executive failure (algorithm + conflict failures; 11/14 possible correlations; 78.5\%). These ranged from a low level, $\rho= \pm .37$ (Stroop test errors, semantic fluency) to a high level, $\rho \geq \pm .49$ (completion time of TMT, MCST series, errors and perseverative errors, Six Elements, Brixton errors). Analyses were also conducted between experimental data and working memory scores (Digit Span subtest of the WAIS-III). Correlations were highlighted between executive failure and the raw score of Digit Span backward only. No correlation was found between measures of working memory and other variables. In addition, no correlation was found between the resolution score of insoluble problems and executive variables. 
TABLE 4

Correlations between cognitive variables and arithmetic protocol scores and comparisons of "deficient" and "nondeficient" patients for soluble-problem solving on cognitive variables

\begin{tabular}{|c|c|c|c|c|c|c|c|c|c|}
\hline \multirow[b]{3}{*}{ Executive functions } & & & & \multirow{3}{*}{$\begin{array}{l}\text { Insoluble } \\
\text { problems } \\
\text { Number of } \\
\text { "solved" }\end{array}$} & \multicolumn{5}{|c|}{ Group comparisons } \\
\hline & \multicolumn{3}{|c|}{ Soluble problems } & & \multicolumn{2}{|c|}{ Deficient patients } & \multicolumn{2}{|c|}{ Nondeficient patients } & \multirow{2}{*}{$\begin{array}{l}\text { Significance } \\
U \text { statistic }\end{array}$} \\
\hline & $\begin{array}{l}\text { Calculation } \\
\text { failure }\end{array}$ & $\begin{array}{l}\text { Executive } \\
\text { failure }\end{array}$ & $\begin{array}{l}\text { Calculation } \\
\text { errors }\end{array}$ & & Mean & $S D$ & Mean & $S D$ & \\
\hline \multicolumn{10}{|l|}{ Inhibition } \\
\hline \multicolumn{10}{|l|}{ Stroop test $(\mathrm{C}-\mathrm{A})$} \\
\hline Time & $.33^{*}$ & $.41 * *$ & $.35^{*}$ & .002 & 153 & 88.5 & 120.5 & 111 & 137.5 \\
\hline $\begin{array}{l}\text { Auto- } \\
\text { corrections }\end{array}$ & .12 & .21 & .17 & .04 & 5.7 & 9.6 & 2.8 & 4.3 & 156 \\
\hline Errors & $.31^{*}$ & $.38^{*}$ & $.41 * *$ & .13 & 8 & 8.2 & 2.7 & 4.5 & $80.5^{* *}$ \\
\hline \multicolumn{10}{|l|}{ MCST } \\
\hline $\begin{array}{l}\text { Perseverative } \\
\text { errors }\end{array}$ & .09 & $.56 * * *$ & .15 & .18 & 9.8 & 5.6 & 5 & 4.6 & $98.5^{* *}$ \\
\hline \multicolumn{10}{|l|}{ Planning } \\
\hline $\begin{array}{l}\text { BADS } 6 \\
\text { Elements }\end{array}$ & $-.57 * * *$ & $-.53^{* * *}$ & $-.57 * * *$ & -.23 & 2.1 & 0.9 & 3.1 & 1.1 & $51^{*}$ \\
\hline \multicolumn{10}{|l|}{ Shifting } \\
\hline \multicolumn{10}{|l|}{ TMT } \\
\hline Time $\mathrm{B}-\mathrm{A}$ & .16 & $.49 * * *$ & .15 & .17 & 266 & 188.8 & 161.5 & 148.2 & $90.5^{*}$ \\
\hline Errors $\mathrm{B}-\mathrm{A}$ & .24 & $.39^{*}$ & $.41 * *$ & -.04 & 1.6 & 0.3 & 0.7 & 0.9 & $97.5^{*}$ \\
\hline \multicolumn{10}{|l|}{ Task coordination } \\
\hline $\begin{array}{l}\text { Dual Task of } \\
\text { Baddeley }(\mu)\end{array}$ & .11 & -.21 & .07 & .22 & 88.5 & 14.1 & 88.6 & 14.8 & 119 \\
\hline \multicolumn{10}{|l|}{ Clustering } \\
\hline \multicolumn{10}{|l|}{ Verbal fluency } \\
\hline $\begin{array}{l}\text { Number of } \\
\text { words }(p)\end{array}$ & $-.30^{*}$ & $-.37 *$ & $-.35^{*}$ & -.01 & 7.5 & 3.75 & 10.8 & 5.8 & 159.5 \\
\hline $\begin{array}{l}\text { Number of } \\
\text { words } \\
\text { (animals) }\end{array}$ & -.05 & $-.38 * *$ & -.13 & -.08 & 13.8 & 6.1 & 18.2 & 10.1 & 161 \\
\hline \multicolumn{10}{|l|}{ Rule detection } \\
\hline \multicolumn{10}{|l|}{ MCST } \\
\hline Series & -.29 & $-.51 * * *$ & $-.29 *$ & -.21 & 3.1 & 1.7 & 4.6 & 1.4 & $97.5^{* *}$ \\
\hline Errors & .15 & $.50 * * *$ & .19 & .25 & 19.7 & 7.1 & 12 & 7 & $92 * *$ \\
\hline \multicolumn{10}{|l|}{ Brixton } \\
\hline Errors & $.32 *$ & $.57 * * *$ & $.35^{*}$ & .15 & 24.4 & 6.6 & 17.1 & 10.2 & $74^{*}$ \\
\hline $\begin{array}{l}\text { Premature } \\
\text { abandons }\end{array}$ & .09 & .22 & .13 & .03 & 1.2 & 1.1 & 0.7 & 1 & 104 \\
\hline \multicolumn{10}{|l|}{ Working memory } \\
\hline $\begin{array}{r}\text { Digit Span } \\
\text { forward }\end{array}$ & -.16 & -.2 & -.15 & .003 & 5.1 & & 5.3 & 0.9 & 123.5 \\
\hline $\begin{array}{l}\text { Digit Span } \\
\text { backward }\end{array}$ & -.11 & $-.49 * * *$ & -.23 & -.30 & 2.9 & & 3.7 & 0.8 & $70 *$ \\
\hline
\end{tabular}

Notes. Spearman rank order correlations were used to study the relationships between cognitive variables and arithmetical scores obtained for all groups of patients. Executive failure score was obtained from the means of algorithm and conflict failures. MannWhitney $U$ tests were used to compare the performances of deficient and nondeficient patients on executive tasks. TMT $=$ Trail Making Test; MCST $=$ Modified Card Sorting Test; BADS $=$ Behavioural Assessment of the Dysexecutive Syndrome.

${ }^{*} p<.05 ;{ }^{* *} p<.01 ;{ }^{* * *} p<.001$.

To refine this analysis, we also formed two subgroups of patients on the basis of the cutoff scores. Those who had a pathological resolution score of soluble problems $(n<5$; number of failures $\geq 8)$ were classified as "deficients," the others (resolution score $\geq 5$; number of failures $\leq 7$ ) as "nondeficients." We conducted comparisons between the two groups on cognitive variables, with nonparametric Mann-Whitney $U$-tests (see Table 4). This analysis confirms the results of correlations, with significant differences for nine criteria (56\%). Differences were higher for measures of inhibition (number of errors in the Stroop test, MannWhitney $U=80.5, p=.003$; MCST perseverative errors, Mann-Whitney $U=98.5, p=.006$ ) and rule detection (MCST series: Mann-Whitney $U=$ 97.5, $p=.005$; MCST errors: Mann-Whitney $U=$ $92, p=.003)$. There were smaller but significant 
differences for planning (Six Elements, MannWhitney $U=51, p=.02$ ), shifting (TMT time completion: Mann-Whitney $U=90.5, p=.02$; TMT errors: Mann-Whitney $U=97.5, p=.04$ ), rule detection (Brixton errors, Mann-Whitney $U=$ $74, p=.02$ ), and working memory (Digit Span backward, Mann-Whitney $U=70, p=.02$ ).

\section{Comparisons of subjects' performances on insoluble problems}

Quantitative analysis. To examine our third hypothesis, a Kruskal-Wallis ANOVA with the between-subject factor group (frontal, subcortical, posterior, control) was run on the number of insoluble problems solved and revealed a significant effect, $H(3,95)=18.07, p<.001$. Frontal patients solved more insoluble problems than subcortical patients, Mann-Whitney $U=124.5$, $p=.04$, posterior patients, Mann-Whitney $U=$ $45, p=.01$, and controls, Mann-Whitney $U=25$, $p=.004$. Differences between other groups were not significant, Mann-Whitney $U$-tests, all $p$ s $>.37$.

Qualitative analysis. We observed four kinds of behavior when participants were confronted to insoluble problems. Table 5 summarizes the behavioral data for each group of participants.

Statistical comparison on number of participants who rejected (rejection + partial resolution) or solved insoluble problems (resolution not argued + reasoned resolution) revealed a significant difference between groups, chi square test, $\chi^{2}(3, N=95)=24.9, p<.001$. This difference is attributable to frontal patients only, who were more numerous in solving insoluble problems than other participants, chi square test, $\chi^{2}(3, N=$ 12) $=18.9, p<.001$. As shown in Table 5 , frontal patients were also the only participants who tried to justify and make plausible responses to insoluble problems (reasoned resolution, $N=8$ ).

\section{Highlighting patient subgroups}

The heterogeneity of patients' performance led us to distinguish subgroups of participants, in order to dissociate different cognitive-behavioral profiles. Using the cutoff scores, we established four distinct subgroups of patients (see Table 6). (a) Patients of Subgroup A performed correctly soluble problems (number of failures $\leq 7$ ) but solved also insoluble items $(n \geq 3)$; (b) patients of Subgroup B failed to solve the soluble problems (number of failures $\geq 8$ ), but rejected insoluble problems $(n \leq 2)$; (c) Subgroup $C$ consisted of subjects who behaved like controls, solving correctly soluble problems (number of failures $\leq 7)$ and rejecting insoluble items $(n \leq 2)$; (d) patients of Subgroup $D$ failed to perform soluble problems (number of failures $\geq 8$ ) and also solved insoluble problems $(n \geq 3)$.

The low number of patients in each subgroup does not allow reliable statistical comparisons. However, only frontal patients showed the four patterns of behavior. More precisely, dissociation was found between members of Subgroups A and B: The first performed soluble problems well while offering solutions to insoluble items; the latter rejected the insoluble problems but showed deficits in performing soluble problems. Figure 1 represents this dissociation, found only in the frontal group, and Appendix B shows examples of problem solving from frontal patients.

\section{DISCUSSION}

The purpose of this study was to establish whether there was a significant relationship between executive functions and environmental dependency, as postulated by Luria and Tsvetkova (1967) and Cummings (1995). This allows us secondly to make some contribution to the issue of the potential link between executive functions and complex problem solving. Third, we want to clarify the semiological aspects of insoluble problem solving, by the

TABLE 5

Types of behavior highlighted during the solving of insoluble problems

\begin{tabular}{|c|c|c|c|c|c|c|c|c|}
\hline \multirow[b]{3}{*}{ Type of behavior } & \multicolumn{8}{|c|}{ Subjects } \\
\hline & \multicolumn{2}{|c|}{ Frontal patients } & \multicolumn{2}{|c|}{ Subcortical patients } & \multicolumn{2}{|c|}{ Posterior patients } & \multicolumn{2}{|c|}{ Controls } \\
\hline & $N$ & $\%$ & $N$ & $\%$ & $N$ & $\%$ & $N$ & $\%$ \\
\hline Rejection & 9 & 45 & 16 & 80 & 10 & 100 & 38 & 84.5 \\
\hline Partial resolution & 2 & 10 & 2 & 10 & 0 & & 6 & 13.3 \\
\hline Resolution not argued $^{\mathrm{a}}$ & 1 & 5 & 2 & 10 & 0 & & 1 & 2.2 \\
\hline Reasoned resolution $^{\mathrm{a}}$ & 8 & 40 & 0 & & 0 & & 0 & \\
\hline
\end{tabular}

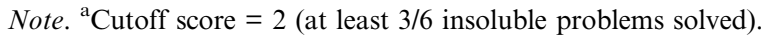


TABLE 6

Arithmetical performances of each subgroup

\begin{tabular}{|c|c|c|c|c|c|c|c|c|c|c|}
\hline \multirow{2}{*}{\multicolumn{2}{|c|}{ Subgroups of patients }} & \multicolumn{3}{|c|}{ Frontal patients } & \multicolumn{3}{|c|}{ Subcortical patients } & \multicolumn{3}{|c|}{ Posterior patients } \\
\hline & & $N$ & Mean & $S D$ & $N$ & Mean & $S D$ & $N$ & Mean & $S D$ \\
\hline \multicolumn{11}{|l|}{ Subgroup A } \\
\hline & Number of subjects & 4 & & & 0 & & & 0 & & \\
\hline & Soluble problems & & 8.5 & 2.5 & & & & & & \\
\hline & Insoluble problems & & 4.8 & 1.5 & & & & & & \\
\hline \multicolumn{11}{|l|}{ Subgroup B } \\
\hline & Number of subjects & 3 & & & 5 & & & 1 & & \\
\hline & Soluble problems & & 3 & 1 & & 2.8 & 1.5 & & 4 & - \\
\hline & Insoluble problems & & 0.3 & 0.6 & & 0.4 & 0.9 & & 0 & - \\
\hline \multicolumn{11}{|l|}{ Subgroup C } \\
\hline & Number of subjects & 8 & & & 13 & & & 9 & & \\
\hline & Soluble problems & & 8.2 & 2.2 & & 7.8 & 2.3 & & 8.5 & 2.5 \\
\hline & Insoluble problems & & 0.1 & 0.3 & & 0.15 & 0.5 & & 0 & \\
\hline \multicolumn{11}{|l|}{ Subgroup D } \\
\hline & Number of subjects & 5 & & & 2 & & & 0 & & \\
\hline & Soluble problems & & 2 & 0.7 & & 3 & 1.4 & & & \\
\hline & Insoluble problems & & 5.8 & 0.4 & & 5.5 & 0.7 & & & \\
\hline
\end{tabular}

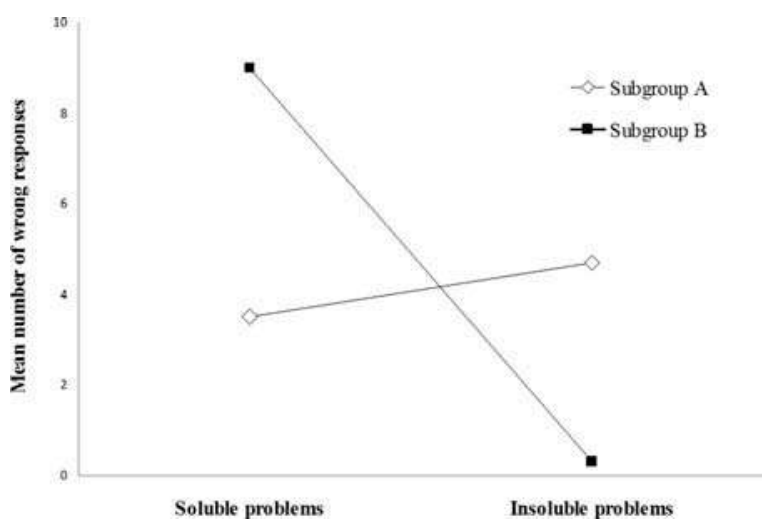

Figure 1. Illustration of the dissociation in the frontal group.

comparison of neurological patients with frontal and nonfrontal brain damage, to determine whether this behavioral disorder is specific to frontal lobe damage and could contribute to the distinction between "dysexecutive" and "frontal" syndromes. We discuss below our two main findings, which are the high association between executive functioning and environmental dependency, and the highlighting of a specific frontal disorder that seems not to be associated with executive functions. This result leads to the proposition of a fractionation of the concept of environmental dependency.

\section{Environmental dependency, executive functions, and soluble problem solving}

The first hypothesis posits an enslavement of the performances of dysexecutive patients to task demands: what Cummings (1995) called "environmental dependency." To explore this assumption, we designed an arithmetic protocol with different levels of algorithm resolution and with conflict problems, in accordance with Luria and Tsvetkova's proposals (1967), and compared the performances of dysexecutive/nondysexecutive patients. If environmental dependency is not linked with executive functions, there should be no difference between the two groups for high level of algorithm resolution and conflict problems. Dysexecutive patients performed significantly worse than nondysexecutive patients in solving soluble problems (total number of problems failed), but a more precise analysis showed that they were impaired only for problems with four operations (Level 3) compared to nondysexecutive. Moreover, the number of conflict problems correctly solved by patients with executive disruption was much lower, approaching the statistical significance $(p=.052)$, whereas there was no difference for the number of nonconflict problems solved. It is noteworthy that the two groups did not differ on the number of problems failed due to calculation error, or the total number of calculation errors. Overall, these results mean that the difference between dysexecutive and nondysexecutive patients on the arithmetic protocol seems attributable only to task demands, irrespective of calculation abilities, confirming our first hypothesis and the theoretical assumption of environmental dependency (Cummings, 1995; Luria \& Tsvetkova, 1967). This interpretation is also corroborated by the fact that patients with executive deficits made significantly more "executive" failures than nondysexecutive patients. 
With regard to clinical implications, these findings have a potential interest for the interpretation of patients' performances during neuropsychological assessment, because it means that impairments on some tasks should be attributable to environmental dependency only. In our study, the qualitative rating (type of failure) permits us to demonstrate that the deficit of some patients was only due to executive failures for high levels of problems (Level 2 and 3) and conflict problems: This is the case for two dysexecutive patients, in both frontal (F19) and subcortical (SC19) groups, who had a resolution score of soluble problems below the cutoff score without making any calculation error. In these cases, the function intended to be evaluated by the task (the ability to make calculations) seems preserved, but the performance in arithmetic problem solving is nevertheless deficient. Our results confirm also the assertions of Domahs, Benke, and Delazer (2011) who have shown recently in a case study that simple calculation (multiplication) can be affected by the structure of the task. Patient's deficits were highlighted only when problems were presented in a list with other operations (e.g., addition, subtraction), while there was no deficit when the same problems were presented in blocked presentation. The authors suggested that an executive impairment could be the cause of the deficit and proposed the term "task-switching acalculia" to define it. They pointed out also the clinical implications of such results for the diagnostic of calculation abilities. In fact, somewhat overlooked in the literature, the concept of environmental dependency must be taken into account for (a) methodological applications in the design of neuropsychological tasks and (b) interpretation of dysexecutive patients' impairments.

Another point of interest in this study was the link between executive functions and complex problem solving, irrespective of the notion of dependency to the task. Several authors have suggested that executive disruption should lead to deficits in complex problem solving (e.g., McNeil \& Burgess, 2002; Semenza et al., 1997; Zamarian et al., 2007; Zamarian et al., 2006), but few comparisons were made between executive and arithmetic data. To contribute to the understanding of this expected relationship and explore the second hypothesis, we focused on the relationship between experimental data and executive variables. Not surprisingly, there were numerous relations between "executive" failures - that is, a failure due to a deficit to plan the resolution algorithm or to inhibit the operation induced by the wording of the task - and executive functions, suggesting that inhibition, shifting, planning, rule detection, and working memory (Digit Span backward) were particularly implicated. These numerous relations seem to validate our rating of these kinds of failures as "executive." However, there were also many relationships between calculation failure/total number of calculation errors and executive data, suggesting an important role of executive functioning in the ability to make simple operations, like addition or subtraction. This result is quite new in the neuropsychological literature, given that the link between executive functions and the ability to make calculations is postulated only for the solving of complex operations like multidigit addition or multidigit multiplication (e.g., McNeil \& Burgess, 2002; Roşca, 2009; Semenza et al., 1997). More specifically, four executive functions seem particularly related with calculation abilities in this study: inhibition, planning, clustering, and rule detection. Future studies should pay attention to the role of executive functions on other numeracy skills not investigated in this work, like multidigit written calculation, approximation, or transcoding - that is, reading, writing, and the transformation of numbers from Arabic or alphabetic formats. Studies in this area of research are still in their infancy, but, for example, Revkin et al. (2008) proposed that cognitive estimation and verbal numerosity estimation depend on executive functioning.

Furthermore, comparisons between "deficient" and "nondeficient" patients on arithmetic problem solving showed that they differed for several executive variables. It is noteworthy that the differences concerned the same executive processes as those highlighted by correlation analyses: inhibition, shifting, planning, rule detection, and also working memory (Digit Span backward). For this latest finding, this is all the more remarkable given that, in this study, there was no difference between patients and controls for measures frequently used to evaluate the central executive of Baddeley's multicomponent model of working memory-that is, Digit Span backward and the dual task of Baddeley. Our results mirrored the literature in that the frequency of deficits on these tasks is generally low (Andres \& Van der Linden, 2002; Baddeley et al., 1997; Vilkki, Levänen, \& Servo, 2002), but it questions also the link between the two major theories of executive functioning. As mentioned above, Baddeley (1986) assumed that the functions of the central executive correspond to an adequate approximation of the processes of the SAS (Norman \& Shallice, 1986). Although we have not evaluated exhaustively the processes depending on the central executive, our findings 
demonstrate that whereas most patients presented a dysexecutive syndrome (74\%), they were spared for some measures of the central executive. Recently, Roussel et al. (2012) have shown that a dysexecutive syndrome is not systematically associated with impaired measures of the central executive. Consequently, the authors argued against the central executive theory of dysexecutive syndrome and proposed that central executive disruption is not the unique cause of executive impairments. To put this assertion in the context of our study, this could mean that the executive interpretation of environmental dependency proposed by Luria and Tsvetkova (1967) seems more appropriate than that of Cummings (1995), in terms of central executive impairment. Overall, our results confirm the proposals of several authors who asserted that inhibition (Zamarian et al., 2007; Zamarian et al., 2006), planning (Semenza et al., 1997), and shifting (McNeil \& Burgess, 2002) may be central processes in complex problem solving.

Nevertheless, some limitations have to be considered. The first concerns the design of the tasks: problems were presented in writing to reduce the charge of working memory, as subjects could read them as many times as desired. However, text comprehension is likely to have an impact on problem solving, insomuch as some publications demonstrated the crucial role of frontal cortex in text comprehension (e.g., Ferstl, Neumann, Bogler, \& von Cramon, 2008; Ferstl \& von Cramon, 2001). More specifically, the semantic comprehension of the text of a problem involves special knowledge about the meaning of specific mathematical terms like "less than" or "more than." This is especially true in the case of conflict problems. Given the fact that we studied patients with posterior brain lesions, who frequently suffer from aphasia and language deficits (word production and comprehension), strict inclusion criteria were established based on the performances on the MT-86 or BDAE aphasia batteries. In addition, a first test with a Level 1 problem was also made before starting the protocol, which allowed the exclusion of patients with impaired understanding. Thus, the interpretation in terms of comprehension deficit seems to be rebuttable in this study.

Second, the definition of the term "complexity" in problem solving is debatable. For example, multidigit addition and multidigit multiplication are deemed more complex than simple operations like those we proposed in this study (e.g., McNeil \& Burgess, 2002; Roşca, 2009; Semenza et al., 1997). So, the question of whether the same executive processes are involved in this kind of complex operation remains to be studied in future works.
This assertion leads us to another point - namely, the link between environmental dependency and complexity. Following the assumption of Luria and Tsvetkova (1967) and the definition proposed by Cummings (1995), we designed a protocol with different structures of problems (with and without conflict, with different levels of algorithm resolution) and have postulated that this modulation would permit the evaluation of environmental dependency. Nevertheless, it seems plausible to argue that this modification of task demands represents also an increase of task complexity, and that the cause of failures can be attributable not only to executive disruption, but also to other factors like general intellectual ability. Nonetheless, our findings give some arguments to the assumption that executive disruption plays a fundamental role in problem solving impairment, given that patients with a dysexecutive syndrome were impaired only when task demands increase, not for simple levels of problems (Levels 1 and 2, nonconflict problems). Moreover, although the structure of problems was different, the operations necessary to solve them were quite similar. So, the difficulty was more in structuring the data to achieve the problem than in reasoning about the best way to solve it - an ability that seems to involve more executive functions than intellectual ability. Furthermore, there was no difference between dysexecutive and nondysexecutive patients for calculation errors and concerning educational level. Nevertheless, this point raises another important question, about the relationship between intelligence and executive functioning. This assumption is a matter of debate, given that some authors stressed the strong relations between intelligence and executive functioning (e.g., Roca et al., 2010). In this publication, we provided some arguments to postulate a strong link between executive functions and environmental dependency, but the hypothesis that intellectual ability plays a key role in environmental dependency should be addressed more precisely by future studies.

\section{Insoluble problem solving and an attempt of theoretical explanation}

The solving of insoluble problems was defined as the inability for frontal patients to challenge the aberrant proposals of the examiner (Aubin et al., 1994; Le Gall et al., 2001; Le Gall et al., 1993). To explore our third hypothesis and test the frontal specificity of this particular kind of behavior, we confronted the resolution score of all participants. Quantitatively, frontal patients solved more 
insoluble problems than other subjects and were also more numerous in demonstrating this behavior $(n=9 ; 45 \%)$. There was no difference between subcortical and posterior patients, who behave like healthy subjects. However, if a majority of subcortical patients and healthy controls rejected immediately insoluble problems (percentage higher or equal to $80 \%$ for each group), some participants have proposed solutions to these items. Thus, according to the pathological threshold (cutoff score), two subcortical patients (10\%) and also one control $(2 \%)$ showed this behavior. In order to specify this result, a qualitative analysis was carried out by distinguishing different kinds of behavior during the solving of insoluble problems. Among the nine frontal patients who solved insoluble problems, eight patients $(89 \%)$ have proposed plausible solutions (reasoned resolution). The means to obtain a number plausible for an age were arbitrary, such as removing a zero to the sum or the selection of the number that most corresponds to an age in the operation. In other words, most frontal patients argued their choice and manipulated the data set to achieve a consistent result, without calling into question the irrelevant proposals of the examiner. The two subcortical patients who solved insoluble problems performed similar operations, but conversely they exerted a critical view, both on the question and on the solutions they proposed. The only control who solved insoluble problems behaves in a similar way, arguing that he made solutions to "satisfy" the examiner. In summary, this is not so much the solutions found by frontal patients that were pathological, but rather their attitude, which seems specific and could be described as a lack of questioning of the experimental material and as an attempt to justify coherently their answer. So, the specific behavior of frontal patients faced by insoluble problems is established both qualitatively and quantitatively. Noteworthy, it concerns almost half of the patients, suggesting that this behavioral disorder is quite frequent in frontal pathology. Given that it remains a clinical fact, we now try to provide a theoretical interpretation to explain this behavior.

First, an overall deficit of reasoning may be thought to be at the origin of the solving of insoluble problems. However, this assertion is undermined by the qualitative analysis of the data. From the threshold values, a dissociation of frontal patients' performances was revealed. Some patients ( $n=4,20 \%$; Subgroup A) did not differ from controls for the resolution score of soluble problems, but proposed responses to insoluble items, while others $(n=3,15 \%$; Subgroup B) showed the opposite profile: They failed to solve soluble problems but rejected insoluble items. This dissociation is highlighted only for the frontal group, which confirms the frontal specificity of the solving of insoluble problems and provides negative answer to the assertion that this behavior may reflect an overall deficit of reasoning. More precisely, the ability to solve a complex arithmetical problem is a multicomponential process that requires different types of knowledge: arithmetical facts (e.g., multiplication tables), procedural knowledge (which guides the execution of algorithms), and the understanding of arithmetic operations and principles concerning these operations - that is, the "conceptual" knowledge (Roşca, 2009). This latter type of knowledge is essential to make inferences and to relate different information involved in arithmetic (Delazer et al., 2004). Given the patterns of performances of frontal patients, it could be assumed that patients from Subgroup B have disturbances in procedural knowledge or arithmetical facts, whereas deficits of patients from Subgroup A are due to an impairment of conceptual knowledge. In this study, we did not assess specifically these different types of knowledge, but the constant concern of patients from Subgroup A to obtain a result that corresponds to a plausible number for an age, as well as their correct solving of soluble problems, seem to demonstrate that conceptual knowledge was spared.

Second, the solving of insoluble problems can be thought to derive from an executive deficit. For example, monitoring capacities have often been associated to frontal regions in various domains (e.g., Fleck, Daselaar, Dobbins, \& Cabeza, 2006). The dissociation reported in the frontal population seems sufficient to contradict this assertion. Indeed, patients from Subgroup A were able to plan the resolution algorithm of complex problems and to inhibit the linear treatment of conflict problems. Moreover, frontal patients who solved insoluble items constantly ensured that their answers matched the question. Thus, the lack of "final control," a deficit of monitoring suggested by Luria and Tsvetkova (1967), does not seem to be an appropriate explanation for these patients. We sought to clarify the executive hypothesis by examining first the relations between executive variables and the resolution score of insoluble problems. We found no relationship between executive data and the resolution score of insoluble problems, and comparisons between dysexecutive and nondysexecutive patients showed that the two groups did not differ on the resolution score of insoluble problems. Moreover, among the six nondysexecutive 
frontal patients, four $(66.6 \%)$ solved insoluble problems, and three frontal patients (75\%) among the four patients of Subgroup A were nondysexecutive. In addition, most of subcortical and posterior patients had a dysexecutive syndrome (respectively $75 \%$ and $80 \%$ ) but they did not attempt to solve insoluble problems. This result is noteworthy because it allows a distinction between "frontal" and "dysexecutive" syndromes, given that (a) the solving of insoluble problems is associated more specifically with frontal brain damage, both in qualitative and in quantitative terms, and (b) there is no association between this behavior and scores on executive tasks.

In the lack of a convincing demonstration of previous hypotheses, we now develop two assumptions that can provide more satisfactory explanations of the solving of insoluble problems in frontal pathology. The first is directly inspired by the works of developmental psychologists, who were the first to explain the behavior of school children who solve insoluble problems. The solving of insoluble problems is well known in developmental psychology literature under the terms of the "ageof-the-captain phenomenon" (e.g., Henn, 2007; Inoue, 2005; Sarrazy, 2002), and the common explanation refers to the children's misinterpretation of the testing situation. This means that insoluble problem solving is not thought to derive from the children's inability to perform correct reasoning, but from their interpretation of the testing situation and of the teacher's expectations. In other words, the performance of school children in problem solving depends not only on their cognitive faculties, but also on their ability to make sense of the situation. This point of view is somewhat overlooked in neuropsychological literature, but Roepstorff and Frith (2004) have stressed that the testing situation requires a shared representation - that is, a common understanding of the objectives of the situation. According to these authors, the formation of such representation is a social process that requires sociocognitive skills, needed to interpret the behavior of others and understand their intentions. The patient/examiner interaction can be seen as an encounter between two social agents who interact around a test designed by one of them (examiner). The other (patient) is not a passive actor, but on the contrary tries to make sense and construct a shared significance of the testing situation. In the example of problem solving, the patient not only has to activate the cognitive abilities necessary to solve the problems, but must also elaborate a social knowledge necessary to apprehend the interaction with the examiner. Confronted with insoluble problems, patients must solve a social problem in addition to an arithmetic problem, because they are left free to interpret the testing situation and choose the attitude they will adopt (propose an answer or not). In this sense, insoluble problems could be considered as "open-ended" tasks. Interestingly, and in accordance with the sociocognitive hypothesis of insoluble problem solving, White (2013; see also White, Burgess, \& Hill, 2009) proposed recently that deficits in open-ended neuropsychological tasks is not attributable to an executive dysfunction, but rather to a sociocognitive deficit, which leads to impairments in forming an understanding of the examiner's expectations.

Second, another recent theory - the false tagging theory (FTT; Asp \& Tranel, 2012) - is likely to provide a meaningful interpretation of the solving of insoluble problems, if we consider this pathological behavior as the result of an inability to call into question the testing situation - that is, to challenge and be skeptical toward the proposals of the examiner. The authors developed a model of belief and doubt that assigns a fundamental role to the prefrontal cortex to affix "false tags" to mental representations initially believed as true. Consequently, the FTT predicts that damage to the prefrontal cortex should lead to a "doubt deficit," which could explain the behavioral and personality traits of prefrontal patients, like gullibility or submissiveness to authority figures (Asp, Ramchandran, \& Tranel, 2012). The solving of insoluble problems seems quite representative of what could happen when "doubt markers" are not affixed to cognitive representation of the task. It is noteworthy that this interpretation could clarify also the frontal specificity of this pathological behavior.

\section{CONCLUSION}

Overall, our findings led us to propose a fractionation of the concept of environmental dependency. Indeed, adopting the point of view of Luria (1979), who stressed the need to take into account qualitative descriptions of patients' behavior in addition to quantitative analysis, we highlighted two forms of environmental dependency during problem solving. The first is characterized by an enslavement of patients' performances to the structure and demands of the task. We confirmed the strong links between this form of dependency and executive disruption, as postulated by Luria and Tsvetkova (1967) and Cummings (1995). Nevertheless, it seems also possible to define a second form of environmental dependency, 
characterized in this study by the tendency to propose an answer to insoluble problems. This second form of dependency seems not to be associated with executive functions and can contribute to the distinction between frontal and dysexecutive syndromes. These preliminary results should be corroborated by tasks other than arithmetic problems, and additional works must be done to confirm that prefrontal patients show specific impairments during open-ended tasks, which necessitate a critical point of view. Nevertheless, whether deficits in these kinds of task are due to a general doubt disruption, as proposed by the FTT (Asp \& Tranel, 2012), or can be explained in sociocognitive terms (e.g., White, 2013) is an important question that future studies should address in order to contribute to a better understanding of prefrontal lobe functions.

\section{REFERENCES}

Allain, P., Verny, C., Aubin, G., Pinon, K., Bonneau, D., Dubas, F., \& Le Gall, D. (2005). Arithmetic word problem solving in Huntington's disease. Brain and Cognition, 57, 1-3.

Anderson, V., Levin, H. S., \& Jacobs, R. (2002). Executive functions after frontal lobe injury: A developmental perspective. In D. Stuss \& V. Anderson (Eds.), Principles of frontal lobe function (pp. 504527). New York, NY: Oxford University Press.

Andres, P., \& Van der Linden, M. (2002). Are central executive functions working in patients with focal frontal lesions? Neuropsychologia, 40, 835-845.

Asp, E. W., Ramchandran, K., \& Tranel, D. (2012). Authoritarianism, religious fundamentalism, and the human prefrontal cortex. Neuropsychology, 26, 414-421.

Asp, E. W., \& Tranel, D. (2012). False tagging theory: Toward a unitary account of prefrontal cortex function. In D. Stuss \& R. T. Knight (Eds.), Principles of frontal lobe function (pp. 383-416). New York, NY: Oxford University Press.

Aubin, G., Le Gall, D., \& Guyard, H. (1994). Etude de la résolution de problèmes numériques chez des patients frontaux [Study of numerical problem solving in patients with frontal lobe lesions]. Revue de Neuropsychologie, 4, 437-467.

Azouvi, P., Didic-Hamel, C. M., Fluchaire, I., Godefroy, O., Hoclet, E., Le Gall, D., ... Pillon, B. (2001). Evaluation des fonctions exécutives en pratique clinique [Assessment of executive functions in clinical practice]. Revue de Neuropsychologie, 11, 383-433.

Baddeley, A. (1986). Working memory. Oxford: Clarendon Press.

Baddeley, A., Della Sala, S., Papagno, C., \& Spinnler, H. (1997). Dual-task performance in dysexecutive and non dysexecutive patients with a frontal lesion. Neuropsychology, 11, 187-194.

Baddeley, A., \& Hitch, G. (1974). Working memory. In G. A. Bower (Ed.), Recent advances in learning and motivation (pp. 47-90). New York, NY: Academic Press.

Beland, R., \& Giroud, F. (1992). Protocole MontréalToulouse d'Examen Linguistique de l'Aphasie [Montreal-Toulouse battery] (Rev. ed.). Isbergues: L'Ortho-Edition.

Bon, L., Belliard, S., Eustache, F., \& Desgranges, B. (2009). L'égocentrisme comportemental dans la démence sémantique: Conséquence d'un trouble de la théorie de l'esprit et/ou de l'égocentrisme cognitif? [Behavioral egocentrism in semantic dementia: Effect of impaired theory of mind and/or cognitive egocentrism?]. Revue de Neuropsychologie, 1, 133-149.

Burgess, P. W., Alderman, N., Volle, E., Benoit, R. G., \& Gilbert, S. J. (2009). Mesulam's frontal lobe mystery re-examined. Restorative Neurology and Neuroscience, 27, 493-506.

Burgess, P. W., \& Shallice, T. (1996). Bizarre responses, rule detection and frontal lobe lesions. Cortex, 32, 241-259.

Campos, S., Zhang, L., Sinclair, E., Tsao, M., Barnes, E. A., Danjoux, C., ... Chow, E. (2009). The palliative performance scale: Examining its inter-rater reliability in an outpatient palliative radiation oncology clinic. Support Care Cancer, 17, 685-690.

Cardebat, D., Doyon, B., Puel, M., Goulet, P., \& Joanette, Y. (1990). Evocation lexicale formelle et sémantique chez des sujets normaux: Performances dynamiques de production en fonction du sexe, de l'âge et du niveau d'étude [Formal and semantic lexical evocation in normal subjects: Dynamic production performance by gender, age and level of education]. Acta Neurologica Belgica, 90, 207-217.

Colette, F., \& van der Linden, M. (2002). Brain imaging of the central executive component of working memory. Neuroscience \& Biobehavioral Reviews, 26, $105-125$.

Cummings, J. L. (1995). Anatomic and behavioral aspects of frontal-subcortical circuits. Annals of the New York Academy of Sciences, 15, 1-13.

Damasio, H., \& Damasio, A. R. (1989). Lesion analysis in neuropsychology. New York, NY: Oxford University Press.

Dehaene, S., \& Cohen, L. (1997). Cerebral pathways for calculation: Double dissociation between rote verbal and quantitative knowledge of arithmetic. Cortex, 33, 219-250.

Delazer, M., Domahs, F., Lochy, A., Karner, E., Benke, T., \& Poewe, W. (2004). Number processing and basal ganglia dysfunction: A single-case study. Neuropsychologia, 42, 1050-1062.

Domahs, F., Benke, T., \& Delazer, M. (2011). A case of "task-switching acalculia." Neurocase, 17, 24-40.

Efron, E. (1987). Better bootstrap confidence intervals. Journal of the American Statistical Association, 82, 171-185.

Efron, E., \& Tibshirani, R. J. (1993). An introduction into the bootstrap. New York, NY: Chapman \& Hall.

Fasotti, L. (1992). Arithmetical word problem solving after frontal lobe damage. A cognitive neuropsychological approach. Amsterdam: Swets \& Zeitlinger.

Ferstl, E. C., Neumann, J., Bogler, C., \& von Cramon, D. Y. (2008). The extended language network: A meta-analysis of neuroimaging studies on text comprehension. Human Brain Mapping, 29, 581-593.

Ferstl, E. C., \& von Cramon, D. Y. (2001). The role of coherence and cohesion in text comprehension: An 
event-related fMRI study. Cognitive Brain Research, $11,325-340$

Fleck, M. S., Daselaar, S. M., Dobbins, I. G., \& Cabeza, R. (2006). Role of prefrontal and anterior cingulate regions in decision-making processes shared by memory and nonmemory tasks. Cerebral Cortex, 16, $1623-1630$.

Folstein, M. F., Folstein, S. E., \& McHugh, P. R. (1975). "Mini-Mental State." A practical method for grading the cognitive state of patients for the clinician. Journal of Psychiatric Research, 12, 189-198.

Godefroy, O. (2003). Frontal syndrome and disorders of executive functions. Journal of Neurology, 250, 1-6.

Godefroy, O., Azouvi, P., Robert, P., Roussel, M., Le Gall, D., \& Meulemans, T. (2010). Dysexecutive syndrome: Diagnostic criteria and validation study. Annals of Neurology, 68, 855-864.

Henn, H. W. (2007). Modelling in school-chances and obstacles. The Montana Mathematics Enthusiast, 3, $125-138$.

Inoue, N. (2005). The realistic reasons behind unrealistic solutions: The role of interpretive activity in word problem solving. Learning and Instruction, 15, 69-83.

Lee, K., Lynn Ng, E., \& Fong Ng, S. (2009). The contributions of working memory and executive functioning to problem representation and solution generation in algebraic word problems. Journal of Educational Psychology, 101, 373-387.

Le Gall, D., Allain, P., \& Aubin, G. (2001). Dissociations et associations dans le syndrome frontal: À propos des troubles de la résolution de problèmes et de l'arrangement de scripts [Dissociations and associations in frontal lobe syndrome: About problem-solving and script sequencing deficits]. Revue de Neuropsychologie, 11, 299-322.

Le Gall, D., Aubin, G., Allain, P., \& Guyard, H. (1993). Résolution de problèmes et manipulation de scripts: Contribution à la compréhension des syndromes frontaux [Problem-solving and script sequencing: Contribution to the understanding of frontal syndromes]. Tétralogiques, 8, 64-104.

Lezak, M. D. (1982). The problem of assessing executive functions. International Journal of Psychology, 17, 281-297.

Lezak, M. D. (1983). Neuropsychological assessment. New York, NY: Oxford University Press.

Luria, A. R. (1966). Higher cortical functions in man. New York, NY: Basic Books.

Luria, A. R. (1979). The making of mind: A personal account of Soviet psychology. Cambridge, MA: Harvard University Press.

Luria, A. R., \& Tsvetkova, L. S. (1967). Les troubles de la résolution de problèmes. Analyse neuropsychologique [Neuropsychological analysis of problem solving]. Paris: Gauthier-Villars.

Mazaux, J. M., \& Orgogozo, J. M. (1981). Boston Diagnostic Aphasia Examination: Échelle Française [French version of the Boston Diagnostic Aphasia Examination]. Issy-les-Moulineaux: Etablissements d'Applications Psychotechniques.

McNeil, J. E., \& Burgess, P. W. (2002). The selective impairment of arithmetical procedures. Cortex, 38, 569-587.

Nelson, H. E. (1976). A modified card sorting test sensitive to frontal lobe defects. Cortex, 12, 313-324.
Norman, D. A., \& Shallice, T. (1986). Attention to action: Willed and automatic control of behavior. In R. J. Davidson, G. E. Schwartz, \& D. Shapiro (Eds.), Consciousness and self-regulation. Advances in research (pp. 1-18). New York, NY: Plenum Press.

Revkin, S. K., Piazza, M., Izard, V., Zamarian, L., Karner, E., \& Delazer, M. (2008). Verbal numerosity estimation deficit in the context of spared semantic representation of numbers: A neuropsychological study of patient with frontal lesions. Neuropsychologia, 46, 2463-2475.

Roca, M., Parr, A., Thompson, R., Woolgar, A., Torralva, T., Antoun, N., ... Duncan, J. (2010). Executive function and fluid intelligence after frontal lobe lesions. Brain, 133, 234-247.

Roepstorff, A., \& Frith, C. (2004). What's at the top in the top-down control of action? Script-sharing and "top-top" control of action in cognitive experiments. Psychological Research, 68, 189-198.

Roşca, E. C. (2009). Arithmetic procedural knowledge: A cortico-subcortical circuit. Brain Research, 1302, $148-156$.

Roussel, M., Dujardin, K., Hénon, H., \& Godefroy, O. (2012). Is the frontal dysexecutive syndrome due to a working memory deficit? Evidence from patients with stroke. Brain, 135, 2192-2201.

Roussel, M., \& Godefroy, O. (2008). La batterie GREFEX: Données normatives [The GREFEX battery: Normative data]. In O. Godefroy \& les membres du GREFEX (Eds.), Fonctions exécutives et pathologies neurologiques et psychiatriques (pp. 231-266). Marseille: Solal.

Samson, S. (2006). Explorations neuropsychologiques de la mémoire chez l'adulte [Neuropsychological examinations of memory in adults]. Epilepsies, 18, 38-45.

Sarrazy, B. (2002). Effects of variability of teaching on responsiveness to the didactic contract in arithmetic problem-solving among pupils of 9-10 years. European Journal of Psychology of Education, 17, 321-341.

Semenza, C., Miceli, L., \& Girelli, L. (1997). A deficit for arithmetical procedures: Lack of knowledge or lack of monitoring? Cortex, 33, 483-498.

Stroop, J. R. (1935). Studies of interference in serial verbal reactions. Journal of Experimental Psychology, 6, 643-661.

Stuss, D. T. (2007). New approaches to prefrontal lobe testing. In B. L. Miller \& J. L. Cummings (Eds.), The human frontal lobe: Functions and disorders (pp. 292305). New York, NY: Guilford Press.

van Harskamp, N. J., \& Cipolotti, L. (2001). Selective impairments for addition, subtraction and multiplication. Implications for the organisation of arithmetical facts. Cortex, 37, 363-388.

Vilkki, J., Levänen, S., \& Servo, A. (2002). Interference in dual-fluency tasks after anterior and posterior cerebral lesions. Neuropsychologia, 40, 340-348.

Wechsler, D. (1997). Wechsler Adult Intelligence Scale (3rd ed.). San Antonio, TX: The Psychological Corporation.

White, S. J. (2013). The triple I hypothesis: Taking another('s) perspective on executive dysfunction in autism. Journal of Autism and Developmental Disorders, 43, 114-121. 
White, S. J., Burgess, P. W., \& Hill, E. L. (2009). Impairments on "open-ended" executive function tests in autism. Autism Research, 2, 138-147.

Wilson, B. A., Alderman, N., Burgess, P. W., Emslie, H., \& Evans, J. (1996). Behavioural Assessment of the Dysexecutive Syndrome. Bury St Edmunds: Thames Valley Test Company.

Zamarian, L., Bodner, T., Revkin, S., Benke, T., Boesch, S., Donnemiller, E., \& Delazer, M. (2009). Numerical deficits in a single case of basal ganglia dysfunction. Neurocase, 15, 390-404.
Zamarian, L., Semenza, C., Domahs, F., Benke, T., \& Delazer, M. (2007). Alzheimer's disease and mild cognitive impairment: Effects of shifting and interference in simple arithmetic. Journal of the Neurological Sciences, 263, 79-88.

Zamarian, L., Visani, P., Delazer, M., Seppi, K., Mair, K. J., Diem, A., ... Benke, T. (2006). Parkinson's disease and arithmetics: The role of executive functions. Journal of the Neurological Sciences, 248, 124-130.

\section{APPENDIX A}

Levels of problems and arithmetic operations needed to solve them

\begin{tabular}{|c|c|c|c|c|}
\hline Number & Level & Number of operations & Description & Operations required to solve the problem \\
\hline 1 & 1 & 2 & non-conflict & $20+(20-5)$ \\
\hline 2 & 2 & 3 & non-conflict & $50+(50-30)+(50+15)$ \\
\hline 3 & - & - & insoluble & - \\
\hline 4 & 3 & 4 & non-conflict & $13+(13-5)+\{[13+(13-5)] \times 3\}$ \\
\hline 5 & 1 & 2 & conflict & $7+(7+2)$ \\
\hline 6 & 2 & 3 & non-conflict & $27+(27-6)+(27 / 3)$ \\
\hline 7 & - & - & insoluble & - \\
\hline 8 & 3 & 4 & conflict & $16+(16+13)+\{[16+(16+13)]-7\}$ \\
\hline 9 & 1 & 2 & non-conflict & $7+(7 \times 5)$ \\
\hline 10 & - & - & insoluble & - \\
\hline 11 & 2 & 3 & conflict & $22+(22+3)+(22-6)$ \\
\hline 12 & 3 & 4 & non-conflict & $19+(19-7)+\{[19+(19-7)]+8\}$ \\
\hline 13 & - & - & insoluble & - \\
\hline 14 & 2 & 3 & conflict & $150+(150-30)+(150-70)$ \\
\hline 15 & 1 & 2 & conflict & $25+(25-12)$ \\
\hline 16 & - & - & insoluble & - \\
\hline 17 & 3 & 4 & conflict & $340+(340-70)+\{[340+(340-70)]-100\}$ \\
\hline 18 & - & - & insoluble & - \\
\hline
\end{tabular}




\section{APPENDIX B}

Examples of executive errors and solving of insoluble problems for frontal patients

Pouvez-vous s'il vous plaît lire ce problème à haute voix et le résoudre en disant ce que vous faites et en écrivant toutes les opérations?

A 12) Dans un bouquet $A$, il y a 19 fleurs. Dans le bouquet $B$, il y a 7 fleurs de moins que dans le bouquet $\mathrm{A}$. Le bouquet $\mathrm{C}$ compte 8 fleurs de plus que les bouquets $\mathrm{A}$ et $\mathrm{B}$ réunis. Combien de fleurs $\mathrm{y}$ a-t-il en tout ?

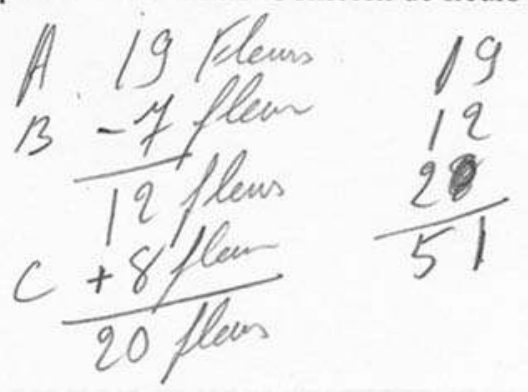

B 14) Un viticulteur Nantais vend 150 bouteilles de vin. Il en vend 30 de plus que le viticulteur Bordelais. Un viticulteur du Jura vend 70 bouteilles de moins que le Nantais. Combien ces trois viticulteurs ont-ils vendus de bouteilles en tout?

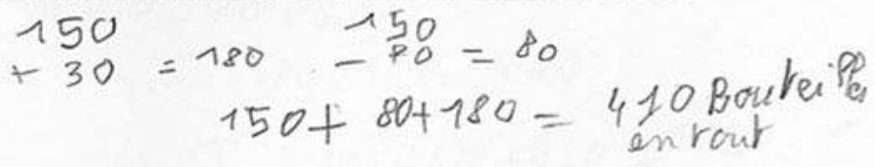

C 10) Sur un bateau, il y a 106 passagers voyageant en seconde classe et 50 en première classe. Quel est l'âge du capitaine?

$$
\begin{aligned}
\text { Age du cafitain } & = \\
106-50 & =56 \text { Ans }
\end{aligned}
$$

D 18) Un fermier vend sur le marché 40 poulets, 240 cufs et 110 litres de lait. Quel est son âge?

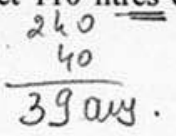

Figure B1. The written instruction was as follows for each problem: "Could you please read aloud this problem and solve it by saying what you do and by writing all operations." (A) Example of an algorithm failure (Patient F13, Subgroup D, Problem Number 12). Order of operations: (a) $19-7=12$; (b) $12+8=20$; (c) $19+12+20=51$. Calculations are correct, but the patient executed the operation $12+8$ instead of $(19+12)+8$, failing to develop the correct resolution algorithm (forgetting an operation). (B) Example of a conflict failure (Patient F11, Subgroup B, Problem Number 14). Order of operations: (a) $150+30=180$; (b) $150-70=80$; (c) $150+80$ $+180=410$. As previously reported by Luria and Tsvetkova (1967), the resolution algorithm and the calculations are correct but the wording of the problem (conflict) induces the wrong operation - the written sentence "Il en vend 30 de plus que le viticulteur bordelais" ("30 more than the Bordeaux winemaker") leads to the operation $150+30$ instead $150-30$. (C) Example of the solving of an insoluble problem (Patient F3, Subgroup A, Problem Number 10). The patient made a subtraction to provide a result correct for an age. (D) Example of the means used for solving a non-plausible insoluble problem (Patient F2, Subgroup D, Problem Number 18). The patient added correctly the data of the problem $(110+240+40=390)$, and given this result was unlikely for an age, she added the letter " $n$ " and "s" to the zero of the sum, achieving a result that corresponds to an age ( 39 ans in French; 39 years old in English). 